\title{
CERTAIN POSITIVE-DEFINITE KERNELS
}

\author{
MINA OSSIANDER AND EDWARD C. WAYMIRE
}

(Communicated by George C. Papanicolaou)

\begin{abstract}
In one way or another, the extension of the standard Brownian motion process $\left\{B_{t}: t \in[0, \infty)\right\}$ to a (Gaussian) random field $\left\{B_{\mathrm{t}}: \mathbf{t} \in \mathbf{R}_{+}^{d}\right\}$ involves a proof of the positive semi-definiteness of the kernel used to generalize $\rho(s, t)=\operatorname{cov}\left(B_{s}, B_{t}\right)=s \wedge t$ to multidimensional time. Simple direct analytical proofs are provided here for the cases of (i) the Lévy multiparameter Brownian motion, (ii) the Chentsov Brownian sheet, and (iii) the multiparameter fractional Brownian field.
\end{abstract}

\section{INTRODUCTION}

The first random field extension of the standard Brownian motion process $\left\{B_{t}: t \in[0, \infty)\right\}$ to "multidimensional time" $\mathbf{x} \in \mathbf{R}^{d}$ is due to Paul Lévy [3], [4]. As defined by Lévy, the multiparameter (standard) Brownian motion is a real-valued Gaussian random field $\left\{B_{\mathbf{x}}: \mathbf{x} \in \mathbf{R}^{d}\right\}$ having mean zero and covariance kernel defined by

$$
\Gamma(\mathbf{x}, \mathbf{y})=\frac{1}{2}\{\|\mathbf{x}\|+\|\mathbf{y}\|-\|\mathbf{x}-\mathbf{y}\|\}, \quad\left(\mathbf{x}, \mathbf{y} \in \mathbf{R}^{d}\right),
$$

where $\|\cdot\|$ denotes the Euclidean norm on $\mathbf{R}^{d}$. The proof of existence of such a random field can proceed from the standard Kolmogorov construction methods once it is shown that the kernel defined by (1.1) is positive semi-definite; i.e., for any $\mathbf{x}_{1}, \ldots, \mathbf{x}_{k} \in \mathbf{R}^{d}, k \geq 1, c_{1}, \ldots, c_{k} \in \mathbf{R}$,

$$
\sum_{1 \leq i, j \leq k} \Gamma\left(\mathbf{x}_{i}, \mathbf{x}_{j}\right) c_{i} c_{j} \geq 0 .
$$

The positive semi-definiteness problem (1.2) for the particular kernel defined by (1.1) had already been solved by Schoenberg [13] a few years prior to Lévy's extension of Brownian motion. In fact, Lévy used Schoenberg's solution in his

Received by the editors February 1, 1989.

1980 Mathematics Subject Classification (1985 Revision). Primary 60G60, 43A35; Secondary $60 \mathrm{G} 15$.

Research by the first author partially sponsored by NSF grant DMS- 8806206 .

Research by the second author partially sponsored by the Army Research Office through grant 26220-GS, the Math. Sciences Institute of Cornell University and by NSF grant DMS-8801466. 
construction; see Lévy [5] for this as well as a systematic account of his results on the sample path regularity of $\left\{B_{\mathbf{x}}\right\}$.

An alternate extension of Brownian motion was introduced by Chentsov [1]. Chentsov's Brownian motion, $\left\{W_{\mathbf{x}}: \mathbf{x} \in \mathbf{R}_{+}^{d}\right\}$, is the mean zero Gaussian random field with covariance kernel defined by

$$
G(\mathbf{x}, \mathbf{y})=\prod_{t=1}^{d} x_{i} \wedge y_{i}, \quad\left(\mathbf{x}=\left(x_{1}, \ldots, x_{d}\right), \mathbf{y}=\left(y_{1}, \ldots, y_{d}\right) \in \mathbf{R}_{+}^{d}\right),
$$

where $a \wedge b=\min \{a, b\}, a, b \in \mathbf{R}$. This process is often referred to as the Brownian sheet. Fundamental distinctions between the Levy and the Chentsov extensions are readily seen by considering the increments. Specifically, defining the partial order $\leq$ on $\mathbf{R}^{d}$ by $\left(x_{1}, \ldots, x_{d}\right)=\mathbf{x} \leq \mathbf{y}=\left(y_{1}, \ldots, y_{d}\right)$ if and only if $x_{i} \leq y_{i}$ for each $i=1, \ldots, d$, one has independence of $W_{\mathrm{y}}-W_{\mathbf{x}}$ and $W_{\mathrm{x}}$ if and only if $\mathbf{x} \leq \mathbf{y}$. In contrast, $B_{\mathbf{y}}-B_{\mathbf{x}}$ and $B_{\mathbf{x}}$ are not independent, unless $\mathbf{x}$ and $\mathbf{y}$ lie along a common ray emanating from the origin. Nonetheless, it is still possible to establish a unifying representation of these two distinct random fields by viewing them as set-indexed processes; see Ossiander and Pyke [10].

A third family of Gaussian random fields arises quite naturally in considerations of extensions of the scaling (or self-similarity) properties of the Brownian field; see Mandelbrot [7]. Namely, to extend the properties $\left\{B_{\lambda \mathbf{x}}\right\} \stackrel{d}{=}\left\{\lambda^{1 / 2} B_{\mathbf{x}}\right\}$, $(\lambda>0\}$, and/or $\left\{W_{\lambda \mathbf{x}}\right\} \stackrel{d}{=}\left\{\lambda^{d / 2} W_{\mathbf{x}}\right\},(\lambda>0)$, where $\stackrel{d}{=}$ denotes equality in distribution, let $\left\{Z_{\mathbf{x}}\right\}$ be the Gaussian random field having zero mean and (for fixed $\beta$ ) covariance kernel defined by

$$
\Gamma_{\beta}(\mathbf{x}, \mathbf{y})=\frac{1}{2}\left\{\|\mathbf{x}\|^{2 \beta}+\|y\|^{2 \beta}-\|\mathbf{x}-\mathbf{y}\|^{2 \beta}\right\} .
$$

Then, provided existence can be established, one has $\left\{Z_{\lambda \mathbf{x}}\right\} \stackrel{d}{=}\left\{\lambda^{\beta} Z_{\mathbf{x}}\right\}$, $(\lambda>0)$. The Gaussian random field $\left\{Z_{\mathbf{x}}\right\}$ with kernel (1.4) is then referred to as the fractional Brownian field with scaling exponent $\beta$. Schoenberg's method for the case $\beta=\frac{1}{2}$ does not extend to the positive semi-definiteness problem with other values of $\beta$. A proof that $\Gamma_{\beta}(\mathbf{x}, \mathbf{y})$ is positive semi-definite for $0 \leq \beta \leq 1$ first appeared as an application of very general results of $\mathrm{R}$. Gangolli [2] on the positive semi-definiteness of kernels of the so-called Lévy-Schoenberg type. In fact the special class of kernels (1.4) provided much of the motivation for Gangolli's general theory. Other proofs are obtained as applications of (relatively deep) white noise integral representations; see for example Ossiander [9], Wong and Zakai [14], Mandelbrot and Van Ness [8] and Pflug [11].

The present paper grew out of our desire to have simple direct solutions of the positive semi-definiteness problem for these kernels. As noted by Gangolli [2], while it is possible that such proofs may be known for these specific kernels, they do not seem to be available in the literature. The solution presented here is obtained by an analysis of a formula suggested by a Poisson approximation proposed by Mandelbrot [6]. This solution is in fact accessible to analysts 
with no familiarity with the probabilistic motivation or applications for this problem.

\section{THE FRACTIONAL BROWNIAN KERNEL}

Lévy's kernel (1.1) is a special case of the fractional kernel (1.4) with $\beta=\frac{1}{2}$. The proof of (1.4) will be established for all $0<\beta<1$. Note that the cases $\beta=0$ and $\beta=1$ may be checked directly as

$$
\sum_{1 \leq i, j \geq k} \Gamma_{0}\left(\mathbf{x}_{i}, \mathbf{x}_{j}\right) c_{i} c_{j}=\sum_{1 \leq i, j \leq k} \frac{1}{2} c_{i} c_{j}=\frac{1}{2}\left(\sum_{i=1}^{k} c_{i}\right)^{2} \geq 0,
$$

and

$$
\begin{aligned}
\sum_{1 \leq i, j \leq k} \Gamma_{1}\left(\mathbf{x}_{i}, \mathbf{x}_{j}\right) c_{i} c_{j} & =\sum_{1 \leq i, j \leq k}\left\langle\mathbf{x}_{i}, \mathbf{x}_{j}\right\rangle c_{i} c_{j}=\left\langle\sum_{i=1}^{k} \mathbf{x}_{i} c_{i}, \sum_{j=1}^{k} \mathbf{x}_{j} c_{j}\right\rangle, \\
& =\left\|\sum_{i=1}^{k} \mathbf{x}_{i} c_{i}\right\|^{2} \geq 0 .
\end{aligned}
$$

For fixed $0<\beta<1$, let

$$
\gamma_{\beta}(s, r)=|s-r|^{\beta-\frac{1}{2}} \operatorname{sign}(s-r)+|r|^{\beta-\frac{1}{2}} \operatorname{sign}(r), \quad s, r \in \mathbf{R}^{1} .
$$

Lemma 2.1. Let $0<\beta<1,-\infty<s, t<\infty$. Then $\gamma_{\beta}(t, \cdot) \in L_{2}\left(\mathbf{R}^{1}, d r\right)$ for each $t$, and

$$
\left\langle\gamma_{\beta}(t, \cdot), \gamma_{\beta}(s, \cdot)\right\rangle=c_{\beta}\left(|t|^{2 \beta}+|s|^{2 \beta}-|t-s|^{2 \beta}\right)
$$

where $c_{\beta}$ is a positive constant and $\langle\cdot, \cdot\rangle$ denotes the usual inner product on $L_{2}\left(\mathbf{R}^{1}, d r\right)$ defined by

$$
\langle f, g\rangle:=\int_{-\infty}^{\infty} f(r) g(r) d r, \quad f, g \in L_{2}\left(\mathbf{R}^{1}, d r\right) .
$$

Proof. First note that $\gamma_{\beta}(0, r)=0$ for all $r$, and for $t \neq 0$.

$$
\gamma_{\beta}^{2}(r, t)=|t|^{2 \beta-1} \cdot \gamma_{\beta}^{2}\left(1, \frac{r}{t}\right),
$$

Now, both $|1-u|^{\beta-\frac{1}{2}} \operatorname{sign}(u)$ and $|u|^{\beta-\frac{1}{2}} \operatorname{sign}(u)$ are locally square integrable in neighborhoods of $u=0$ and $u=1$, so $\gamma_{\beta}^{2}(1, u)$ is as well. Moreover, for $\beta<1$,

$$
\begin{aligned}
& \int_{-\infty}^{-2} \gamma_{\beta}^{2}(1, u) d u+\int_{2}^{\infty} \gamma_{\beta}^{2}(1, u) d u \\
& \quad=2 \int_{2}^{\infty} \gamma_{\beta}^{2}(1, u) d u=2 \int_{2}^{\infty}\left[u^{\beta-\frac{1}{2}}-(u-1)^{\beta-\frac{1}{2}}\right]^{2} d u \\
& \quad=2 \int_{2}^{\infty}\left(\int_{u-1}^{u}\left(\beta-\frac{1}{2}\right) \nu^{\beta-\frac{3}{2}} d \nu\right)^{2} d u \leq 2\left(\beta-\frac{1}{2}\right)^{2} \int_{2}^{\infty} \int_{u-1}^{u} \nu^{2 \beta-3} d \nu d u \\
& \quad \leq 2\left(\beta-\frac{1}{2}\right)^{2} \int_{2}^{\infty}(u-1)^{2 \beta-3} d u<\infty .
\end{aligned}
$$


Thus, $\gamma_{\beta}(t, \cdot) \in L_{2}\left(\mathbf{R}^{1}, d r\right)$ for each $t$ and by $(2.5)$ we have $\left\|\gamma_{\beta}(t, \cdot)\right\|_{2}^{2}=$ $2 c_{\beta}|t|^{2 \beta}$ where $2 c_{\beta}:=\left\|\gamma_{\beta}(1, \cdot)\right\|_{2}^{2}>0$. By linearity of $L_{2}\left(\mathbf{R}^{1}, d r\right)$, it also follows that $\gamma_{\beta}(t, \cdot)-\gamma_{\beta}(s, \cdot) \in L_{2}\left(\mathbf{R}^{1}, d r\right)$. Moreover

$$
\begin{aligned}
\left\|\gamma_{\beta}(t, \cdot)-\gamma_{\beta}(s, \cdot)\right\|_{2}^{2} & =\int_{-\infty}^{\infty}\left\{|t-r|^{\beta-\frac{1}{2}} \operatorname{sign}(t-r)-|s-r|^{\beta-\frac{1}{2}} \operatorname{sign}(s-r)\right\}^{2} d r \\
& =\int_{-\infty}^{\infty}\left\{|t-s+x|^{\beta-\frac{1}{2}} \operatorname{sign}(t-s+x)-|x|^{\beta-\frac{1}{2}} \operatorname{sign}(x)\right\}^{2} d x \\
& =\int_{-\infty}^{\infty}\left\{|t-s-y|^{\beta-\frac{1}{2}} \operatorname{sign}(t-s-y)+|y|^{\beta-\frac{1}{2}} \operatorname{sign}(y)\right\}^{2} d y \\
& =\left\|\gamma_{\beta}(t-s, \cdot)\right\|_{2}^{2}=2 c_{\beta}|t-s|^{2 \beta} .
\end{aligned}
$$

Thus, the result follows from bilinearity of the inner product as expressed in the simple identity

$$
\left\langle\gamma_{\beta}(t, \cdot), \gamma_{\beta}(s, \cdot)\right\rangle=\frac{1}{2}\left\{\left\|\gamma_{\beta}(t, \cdot)\right\|_{2}^{2}+\left\|\gamma_{\beta}(s, \cdot)\right\|_{2}^{2}-\left\|\gamma_{\beta}(t, \cdot)-\gamma_{\beta}(s, \cdot)\right\|_{2}^{2}\right\} .
$$

Theorem 2.1. The kernel $\Gamma_{\beta}(\mathbf{x}, \mathbf{y})$ is positive semi-definite for each $0<\beta<1$. Proof. For each $d \geq 1, \mathbf{x}, \mathbf{y} \in \mathbf{R}^{d}, \boldsymbol{\theta} \in S^{d-1}:=\left\{\boldsymbol{\theta} \in \mathbf{R}^{d}:\|\boldsymbol{\theta}\|=1\right\}$ we have, by $(2.4)$,

$$
\begin{aligned}
\int_{S^{d-1}} & \int_{-\infty}^{\infty} \gamma_{\beta}(\langle\mathbf{x}, \boldsymbol{\theta}\rangle, r) \gamma_{\beta}(\langle\mathbf{y}, \boldsymbol{\theta}\rangle, r) d r d \boldsymbol{\theta} \\
= & \int_{S^{d-1}} \frac{1}{2} c_{\beta}\left\{|\langle\mathbf{x}, \boldsymbol{\theta}\rangle|^{2 \beta}+|\langle\mathbf{y}, \boldsymbol{\theta}\rangle|^{2 \beta}-|\langle\mathbf{x}-\mathbf{y}, \boldsymbol{\theta}\rangle|^{2 \beta}\right\} d \boldsymbol{\theta} \\
= & C_{d, \beta}\left\{\|\mathbf{x}\|^{2 \beta}+\|y\|^{2 \beta}-\|\mathbf{x}-\mathbf{y}\|^{2 \beta}\right\},
\end{aligned}
$$

where

$$
C_{d, \beta}=\frac{1}{2} c_{\beta} \int_{S^{d-1}}|\langle\varphi, \theta\rangle|^{2 \beta} d \theta
$$

does not depend on $\varphi \in S^{d-1}$. Here $\langle\mathbf{x}, \boldsymbol{\theta}\rangle=\sum_{i=1}^{d} x_{i} \theta_{i}$ is the ordinary dot product and $d \boldsymbol{\theta}$ is normalized surface area (Haar measure) on $S^{d-1}$. Now simply note that for any $\mathbf{x}_{1}, \ldots, \mathbf{x}_{\kappa} \in \mathbf{R}^{d}, c_{1}, \ldots, c_{\kappa} \in \mathbf{R}$,

$$
\begin{aligned}
\sum_{1 \leq i, j \leq \kappa} \Gamma_{\beta}\left(\mathbf{x}_{i}, \mathbf{x}_{j}\right) c_{i} c_{j} & =\sum_{1 \leq i, j \leq \kappa} c_{i} c_{j} \int_{S^{d-1}} \int_{-\infty}^{\infty} \gamma_{\beta}\left(\left\langle\mathbf{x}_{i}, \boldsymbol{\theta}\right\rangle, r\right) \gamma_{\beta}\left(\left\langle\mathbf{x}_{j}, \boldsymbol{\theta}\right\rangle, r\right) d r d \theta \\
& =\int_{S^{d-1}} \int_{-\infty}^{\infty}\left(\sum_{i=1}^{\kappa} c_{i} \gamma_{\beta}\left(\left\langle\mathbf{x}_{i}, \boldsymbol{\theta}\right\rangle, r\right)\right)^{2} d r d \boldsymbol{\theta} \geq 0 .
\end{aligned}
$$

\section{The BRowniAN SHEET}

Our approach to the proof of the positive semi-definiteness for the kernel (1.3) is to let $\chi=\max \left\{x_{i}^{(\nu)}: i=1, \ldots, k, \nu=1, \ldots, d\right\}$ for $\mathbf{x}_{1}, \ldots, \mathbf{x}_{k} \in \mathbf{R}_{+}^{d}$ 
and consider the compact operator $T$ on $L_{2}[0, \chi]$ defined by

$$
T f(x)=\int_{0}^{\chi} f(y)(x \wedge y) d y, \quad(0 \leq x \leq \chi) .
$$

Since $x \wedge y=y \wedge x, T$ is clearly self-adjoint. It follows from the HilbertSchmidt Theorem [12, Thm. V1.16] that there is a complete orthonormal basis $\left\{\varphi_{n}\right\}$ for $L_{2}[0, \chi]$ consisting of eigenfunctions of $T$ with corresponding positive eigenvalues $\left\{\lambda_{n}\right\}$. Thus,

$$
x \wedge y=\sum_{n=1}^{\infty} \lambda_{n} \varphi_{n}(x) \varphi_{n}(y), \quad 0 \leq x, y \leq \chi .
$$

Theorem 3.1. The kernel $G(\mathbf{x}, \mathbf{y})=\prod_{i=1}^{d} x_{i} \wedge y_{i}, \mathbf{x}, \mathbf{y} \in \mathbf{R}_{+}^{d}$ is positive semidefinite.

Proof. For $\mathbf{x}_{1}, \ldots, \mathbf{x}_{\kappa} \in \mathbf{R}_{+}^{d}, c_{1}, \ldots, c_{\kappa} \in \mathbf{R}$ we obtain from an eigenfunction expansion of the form (3.2) that

$$
\begin{aligned}
& \sum_{1 \leq i, j \leq \kappa} G\left(\mathbf{x}_{i}, \mathbf{x}_{j}\right) c_{i} c_{j} \\
& =\sum_{1 \leq i, j \leq \kappa} c_{i} c_{j} \prod_{\nu=1}^{d} \sum_{n_{\nu}=1}^{\infty} \lambda_{n_{\nu}} \varphi_{n_{\nu}}\left(x_{i}^{(\nu)}\right) \varphi_{n_{\nu}}\left(x_{j}^{(\nu)}\right) \\
& \quad=\sum_{n_{1}=1}^{\infty} \ldots \sum_{n_{\kappa}=1}^{\infty} \lambda_{n_{1}} \ldots \lambda_{n_{\kappa}} \sum_{1 \leq i, j \leq \kappa} c_{i} c_{j} \varphi_{n_{1}}\left(x_{i}^{(1)}\right) \ldots \varphi_{n_{d}}\left(x_{i}^{(d)}\right) \\
& \quad \times \varphi_{n_{1}}\left(x_{j}^{(1)}\right) \ldots \varphi_{n_{d}}\left(x_{j}^{(d)}\right) \\
& \quad=\sum_{n_{1}=1}^{\infty} \ldots \sum_{n_{\kappa}=1}^{\infty} \lambda_{n_{1}} \ldots \lambda_{n_{\kappa}}\left(\sum_{i=1}^{\kappa} c_{i} \varphi_{n_{1}}\left(x_{i}^{(1)}\right) \ldots \varphi_{n_{d}}\left(x_{i}^{(d)}\right)\right)^{2} \geq 0
\end{aligned}
$$

since $\lambda_{n}>0$ for all $n=1,2, \ldots$.

\section{REFERENCES}

1. N. N. Chentsov, Weiner random fields depending on several parameters, Dokl. Akad. Nauk SSSR (N.S.) 106 (1956), 607-609.

2. R. Gangolli, Positive definite kernels on homogeneous spaces and certain stochastic processes related to Lévy's Brownian motion of several parameters, Ann. Inst. Henri Poincaré 3, Série B, (1967), 121-225.

3. P. Lévy, Le mouvement Brownien plan, Amer. J. Math. 62 (1940), 487-550.

4. $\ldots$ Sur le mouvement brownien dépendant de plusieurs parametres, C. R. Acad. Sci. Paris 220 (1945), 420-422.

5. __ Processus stochastiques et mouvement brownien, Gauthier Villars, Paris, 1948.

6. B. B. Mandelbrot, Fonctions aléatoires pluri-temporelles poissonienne du cas brownien et fénéralisations, C. R. Acad. Sc. Paris 280A (1975), 1075-1078.

7. _ The fractal geometry of nature, W. H. Freeman and Co., San Francisco, 1982.

8. B. B. Mandelbrot and J. Van Ness, Fractional Brownian motions, fractional noises and applications, SIAM Review 10 (1968), 422-437. 
9. M. Ossiander, Weak convergence and a law of the iterated logarithm for partial-sum processes indexed by points in a metric space, University of Washington, Ph.D. dissertation, 1984.

10. M. Ossiander and R. Pyke, Lévy's Brownian motion as a set-indexed process and a related central limit theorem, Stoch. Process. Appl. 21 (1985), 133-145.

11. G. Pflug, A statistically important Gaussian process, Stoch. Process. Appl. 13 (1982), 45-57.

12. M. Reed and B. Simon, Methods of modern mathematical physics, vol. 1, Academic Press, New York, 1980.

13. I. J. Schoenberg, Metric spaces and positive definite functions, Trans. Amer. Math. Soc. 44 (1938), 522-536.

14. E. Wong and M. Zakai, Martingales and stochastic integrals for processes with a multidimensional parameter, Z. Wahrsch. verw. Gebiete 29 (1974), 109-122.

Department of Mathematics, Oregon State University, Corvallis, Oregon 97331 